\title{
DEF6 expression in ovarian carcinoma correlates with poor patient survival
}

\author{
Phui-Ly Liew ${ }^{1,2}$, Chih-Yeu Fang ${ }^{2,3}$, Yu-Chieh Lee ${ }^{4}$, Yi-Chih Lee ${ }^{5}$, Chi-Long Chen ${ }^{2,6^{*}}$ and Jan-Show Chu ${ }^{2,6^{*}}$
}

\begin{abstract}
Background: Increased expression of DEF6 is correlated with the malignant behavior of various cancers. Both DEF6 and p16 contribute to the regulation of cell cycle progression, and p53 plays important role in the cell cycle checkpoints. This study was designed to elucidate the prognostic significance of DEF6, p53 and p16 immunoexpressions in different histology subtypes of ovarian carcinoma.

Methods: Immunohistochemistry results of DEF6, p53 and p16 on ovarian carcinoma were compared with histology subtypes, clinical data, overall survival (OS) and disease-free survival (DFS) by Cox regression and Kaplan-Meier analysis.

Results: We studied 180 cases of ovarian carcinomas (75 high-grade serous, 41 clear cell, 36 mucinous and 28 endometrioid), including $109 \mathrm{FIGO}$ stage I-II cases and 71 FIGO stage III-IV cases. Ovarian carcinomas positive for both DEF6 and p16 expression were associated with the worst OS $(P=0.027)$ and DFS $(P=0.023)$, whereas those negative for both DEF6 and p16 had the best OS and DFS. Aberrant p53 expression combined with positive DEF6 was associated with worst OS $(P=0.031)$ and DFS $(P=0.028)$. Kaplan-Meier analysis showed that significantly shorter survival rates were seen in patients with high expressions of DEF6 $(P=0.008)$ and p16 $(P=0.022)$. Patients with aberrant p53 expression in highgrade serous carcinoma $(P=0.012)$ and patients with high DEF6 expression in clear cell carcinoma $(P=0.001)$ were also associated with shorter overall survival. In univariate analysis, FIGO stage, DEF6 and p16 were associated with poor prognosis. DEF6 expression was the only independent prognostic factor correlated with shorted OS (HR 2.115; $P=0.025$ ) and DFS (HR 2.248; $P=0.016$ ) upon multivariate analysis.
\end{abstract}

Conclusions: DEF6 expression may serve as an independent prognostic factor, and interacted positively with p16 toward high tumor stage and shorter survival.

Keywords: Ovarian carcinoma, DEF6, p16, Prognosis

\section{Background}

Ovarian carcinoma is the most lethal malignancy of the female genital tract, mainly due to the failure of early diagnosis, heterogeneous histology subtypes and the limitations for the conventional chemotherapies $[1,2]$. The important prognostic factors include tumor stage, age at initial diagnosis, tumor morphological subtypes and grade, optimal resection for advanced ovarian cancer, as well as the effect of chemotherapy following primary surgery [3]. Current researches have focused on the study of various molecular signaling reactions or pathways in ovarian carcinoma to explore the molecular

\footnotetext{
* Correspondence: chencl@tmu.edu.tw; jschu@tmu.edu.tw

${ }^{2}$ Department of Pathology, School of Medicine, College of Medicine, Taipei

Medical University, No. 250, Wu Xing Street, Taipei 11031, Taiwan

Full list of author information is available at the end of the article
}

markers for early detection, prognosis assessment and hopefully as potential therapeutic targets.

Interferon regulatory factor 4 binding protein (IBP, also known as: DEF6) first identified in 2003 [4], plays multiple important roles in various biological processes that involve the immune system. Loss of DEF6 in mice resulted in the development of systemic autoimmunity and developmental defects at the earliest stage of thymocyte differentiation [5-10]. Besides, DEF6 plays important role in the regulation of cell motility, cytoskeletal rearrangements, focal complex/adhesion assembly, cell polarity and cell migration through the stimulation of actin polymerization [11-17].

Signaling involving DEF6 has been implicated in tumorigenesis. Increased expression of DEF6 has been shown to be correlated with the malignant behavior of 
extra-skeletal myxoid chondrosarcoma [18], colorectal cancer [19], breast cancer cells [20], and oral squamous cell carcinoma [21]. DEF6 may serve as a potential target for anti-angiogenic intervention in renal cell carcinoma [22]. DEF6 is also a novel target of tumor suppressor p53 and can suppress cisplatin-mediated apoptosis of breast cancer cells via a negative feedback regulation of the p53 signaling pathway [23]. High levels of DEF6 were found to decrease cisplatin-induced growth suppression and apoptotic cell death, in association with decreased p53 activity and imbalanced expressions of the Bcl-2 family members. Recently, p16 has been shown as a prognostic indicator in ovarian/fallopian tubal highgrade serous carcinoma [24-26]. Moreover, both DEF6 and p16 contribute to the regulation of cell cycle progression, and p53 plays important role in the cell cycle checkpoints. Despite their potential close interaction in cell cycle progression, the roles of DEF6, p16 and p53 have not been fully elucidated in ovarian carcinomas.

In the present study, we sought to better understand the differential expression of DEF6, its relation to the expressions of p53 and p16, and the prognostic significance in different histology subtypes of ovarian carcinoma based on a well-defined cohort of ovarian carcinomas. Clinicopathological data and survival curves were compared between patients with different scores of DEF6 to explore the potential of DEF6 as a prognostic marker.

\section{Methods}

\section{Patient specimens}

We collected cases with surgically operated ovarian carcinomas from the files of Departments of Pathology of Taipei Medical University Hospital and Wan Fang Medical Center between January 1998 and December 2011. We used the diagnostic criteria of the World Health Organization classification [1] and tumor staging system of the International Federation of Gynecology and Obstetrics (FIGO). The pathological diagnosis was reviewed by at least two pathologists. Cases of secondary metastasis to the ovaries were excluded.

Pathologic variables included histology subtypes (i.e. low- or high-grade serous carcinoma, mucinous carcinoma, endometrioid carcinoma and clear cell carcinoma), stage, therapy, recurrence free interval (if applicable), and site of recurrent disease (if applicable). Debulking surgery was found to be optimal if the maximum diameters of the individual residual tumor deposits were all less than $1.0 \mathrm{~cm}$. Only cases with optimal resection were enrolled and analyzed in this study. Patient information were de-identified and assigned a study number. Tissue microarrays (TMA) with three to four tumor regions were chosen from paraffin embedded blocks. Each tumor core measured $0.3 \mathrm{~cm}$ in maximal diameter. This study had been reviewed and approved by the Institutional Review Board of Taipei Medical University (TMU-IRB 99049).

\section{Immunohistochemistry and interpretation}

The DEF6 monoclonal antibody (clone 1 F2; Abnova, Taiwan; 1: 3000), p53 (clone: DO-7; Ventena; prediluted), and p16 (clone: E6H4; Ventena; prediluted) were used. Formalin-fixed and paraffin-embedded tissue sections were cut, deparaffinised and rehydrated. Autoclaved retrieval technique by using $10 \mathrm{mM}$ citric acid buffer (10-20 min) and inhibited by endogenous peroxidase activity $(0.3 \%$ $\mathrm{H}_{2} \mathrm{O}_{2} ; 5 \mathrm{~min}$ ) were conducted. Tissue sections were incubated with primary antibodies in an automated stainer system (Ventana, BenchMark XT). Tissue sections were then incubated with secondary antibody (dilution rate 1:100, $30 \mathrm{~min})$, peroxidase-conjugated streptavidin $(100 \mu \mathrm{g} / \mathrm{mL})$, and $0.02 \%$ 3,3'-diaminobenzidine tetrahydrochloride (DAB) $\left(0.05 \mathrm{M}\right.$ Tris- $\mathrm{HCl}$ buffer with $\left.0.03 \% \mathrm{H}_{2} \mathrm{O}_{2}\right)$. All slides were counterstained with hematoxylin and analyzed by two pathologists (P-L Liew and C-L Chen).

The DEF6 cytoplasmic staining results were scored according to the percentages of positive cells: score 0 (less than $5 \%)$, score 1 (5-24\%), score 2 (25-75\%), and score 3 (more than $75 \%$ ). The stromal lymphocytes were served as positive internal control. The results of p53 and p16 immunostains were scored according to the percentages of positive cells: score $0(0 \%)$, score 1 (1$24 \%$ ), score 2 (25-75\%), and score 3 (more than $75 \%$ ). Score 0 and score 3 of p53 were recorded as aberrant expression; whereas score 1 and score 2 of p53 were designated as insignificant expression.

\section{Cell lines and cell lysates}

Total cell lysates from six ovarian carcinoma cells, including A2780, ES-2, TOV-21G, TOV-112D, OVCAR3, and HBT75, were used for the detection of DEF6 expression by Western blotting. A2780 (ovarian carcinoma cell line with unknown disease type identification) cell was cultured in RPMI-1640 with $10 \%$ fetal bovine serum (FBS). ES-2 (clear cell carcinoma), TOV-21G (clear cell carcinoma) and TOV-112D (endometrioid carcinoma) were obtained from ATCC bioresource center (Manassas, VA, USA). ES-2 was cultured in McCoy's 5 medium with $10 \%$ FBS. TOV-21G and TOV-112D were cultured in a 50:50 mixture of Medium 199 and MCDB105 with $10 \%$ FBS. Cells were grown to near confluent, washed with PBS, and then lysed by RIPA lysis buffer. The lysates of OVCAR3 (serous carcinoma) and HBT75 (serous carcinoma) cells were kindly provided by Prof. Chao-Lien Liu at the Department of Medical Laboratory Science and Biotechnology, Taipei Medical University. 


\section{Western blot assay}

Lysates from the six ovarian carcinoma cell lines and two positive control oral carcinoma cells, HSC-3 and SCC25, were separated in a $10 \%$ polyacrylamide gel and transferred onto a PVDF membrane. After blocking, the blot was incubated with indicated antibodies overnight at $4{ }^{\circ} \mathrm{C}$. Antibodies against DEF6and $\beta$-actin (Genetex, Irvine, CA, USA) were used as the primary antibodies. After washing, the blot was incubated with horseradish peroxidaselabeled goat anti-mouse/rabbit IgG (Jackson Laboratory). The expression profile of the proteins was visualized using a Western Lightening-ECL kit (PerkinElmer, Waltham, MA, USA).

\section{Statistical analyses}

We performed statistical analyses by using SPSS for Windows software (SPSS, Chicago, IL). Data were expressed as median (interquartile range), mean (SD), and percentages. Chi-square test, Mann-Whitney U tests, and Student's $t$-test were used as statistical methods. Spearman rank correlation and multivariate linear regressions with stepwise variable selection were performed to assess the significant associations between ordinal or continuous predictor variables. The KaplanMeier method, Cox proportional hazard regression model and multifactorial Cox regression analysis were used to examine all factors found to be prognostic of survival in univariate analysis and the analysis of DFS and OS. A P-value of less than 0.05 was considered statistically significance.

\section{Results}

Comparison of DEF6, p53 and p16 expressions in different histological subtypes

In our cohort of study, we collected 180 cases of ovarian carcinomas (75 high-grade serous, 41 clear cell, 36 mucinous, and 28 endometrioid carcinomas). We did not enroll cases of low-grade serous carcinoma because only three cases of low-grade serous carcinoma were diagnosed. The patients' ages ranged from 25 to 93 years (mean: 53.8 years). Totally 109 early stage cases (FIGO stage I-II) and 71 advanced stage (FIGO stage III-IV), with a median follow-up time of 33.0 months (mean, 48.3 months; range, 1 to 146 months) were enrolled. The surgical procedures included total hysterectomy, bilateral salpingo-oophorectomy, pelvic and/or para-aortic lymph nodes sampling and omentectomy.

As shown in Table 1, immunoexpressions of DEF6, p53 and p16 showed significant correlation with different histological subtypes of ovarian carcinoma. Score 3 immunoexpression of DEF6 could be assessed in $52 \%$ $(39 / 75)$ and $64.3 \%(18 / 28)$ of high-grade serous carcinoma (Figs. 1a, b, c and d) and endometrioid carcinoma, respectively. Aberrant p53 expression was also observed in $82.7 \%$ of high-grade serous carcinoma. The p16 expression was either cytoplasmic or showed a combination of nuclear and cytoplasmic immunoreactivity. The

Table 1 Correlation of DEF6, p53 and p16 expression in histological subtypes of ovarian carcinoma

\begin{tabular}{|c|c|c|c|c|c|c|c|c|c|c|}
\hline \multirow[b]{3}{*}{ Antibody } & \multirow[b]{3}{*}{ Score } & \multicolumn{9}{|c|}{ Histology subtypes of ovarian carcinoma } \\
\hline & & \multicolumn{2}{|c|}{ Serous carcinoma $(N=75)$} & \multicolumn{2}{|c|}{ Mucinous carcinoma $(N=36)$} & \multicolumn{2}{|c|}{ Endometrioid carcinoma $(N=28)$} & \multicolumn{3}{|c|}{ Clear cell carcinoma $(N=41)$} \\
\hline & & No. & (\%) & No. & (\%) & No. & (\%) & No. & (\%) & $P$-value \\
\hline \multirow[t]{5}{*}{ DEF6 } & 0 & 8 & $(10.7)$ & 8 & $(22.2)$ & 2 & $(7.1)$ & 6 & $(14.6)$ & $<0.001^{*}$ \\
\hline & 1 & 7 & (9.3) & 12 & (33.3) & 4 & $(14.3)$ & 15 & (36.6) & \\
\hline & 2 & 21 & $(28.0)$ & 9 & $(25.0)$ & 4 & $(14.3)$ & 11 & $(26.8)$ & \\
\hline & 3 & 39 & $(52.0)$ & 7 & $(19.4)$ & 18 & $(64.3)$ & 9 & $(22.0)$ & \\
\hline & NA & 0 & $(0.0)$ & 0 & $(0.0)$ & 0 & $(0.0)$ & 0 & $(0.0)$ & \\
\hline \multirow[t]{5}{*}{ p53 } & 0 & 26 & (34.7) & 12 & (33.3) & 6 & (21.4) & 6 & (14.6) & $<0.001^{*}$ \\
\hline & 1 & 11 & $(14.7)$ & 17 & $(47.2)$ & 18 & (64.3) & 29 & (70.7) & \\
\hline & 2 & 1 & (1.3) & 2 & (5.6) & 1 & (3.6) & 2 & (4.9) & \\
\hline & 3 & 36 & (48.0) & 5 & (13.9) & 3 & (10.7) & 4 & (9.8) & \\
\hline & NA & 1 & (1.3) & 0 & $(0.0)$ & 0 & $(0.0)$ & 0 & $(0.0)$ & \\
\hline \multirow[t]{5}{*}{ p16 } & 0 & 12 & (16.0) & 29 & (80.6) & 11 & (39.3) & 14 & (34.1) & $<0.001^{*}$ \\
\hline & 1 & 6 & (8.0) & 3 & (8.3) & 6 & $(21.4)$ & 16 & (39.0) & \\
\hline & 2 & 0 & $(0.0)$ & 0 & (0.0) & 0 & $(0.0)$ & 0 & $(0.0)$ & \\
\hline & 3 & 56 & $(74.7)$ & 3 & (8.3) & 11 & (39.3) & 10 & (24.4) & \\
\hline & NA & 1 & (1.3) & 1 & (2.8) & 0 & $(0.0)$ & 1 & (2.4) & \\
\hline
\end{tabular}

NA, Not applicable (not enough material for analysis or technical limitations)

$P$ - value Fisher's exact or Chi-square test analysis ( ${ }^{*}$ significant difference) 

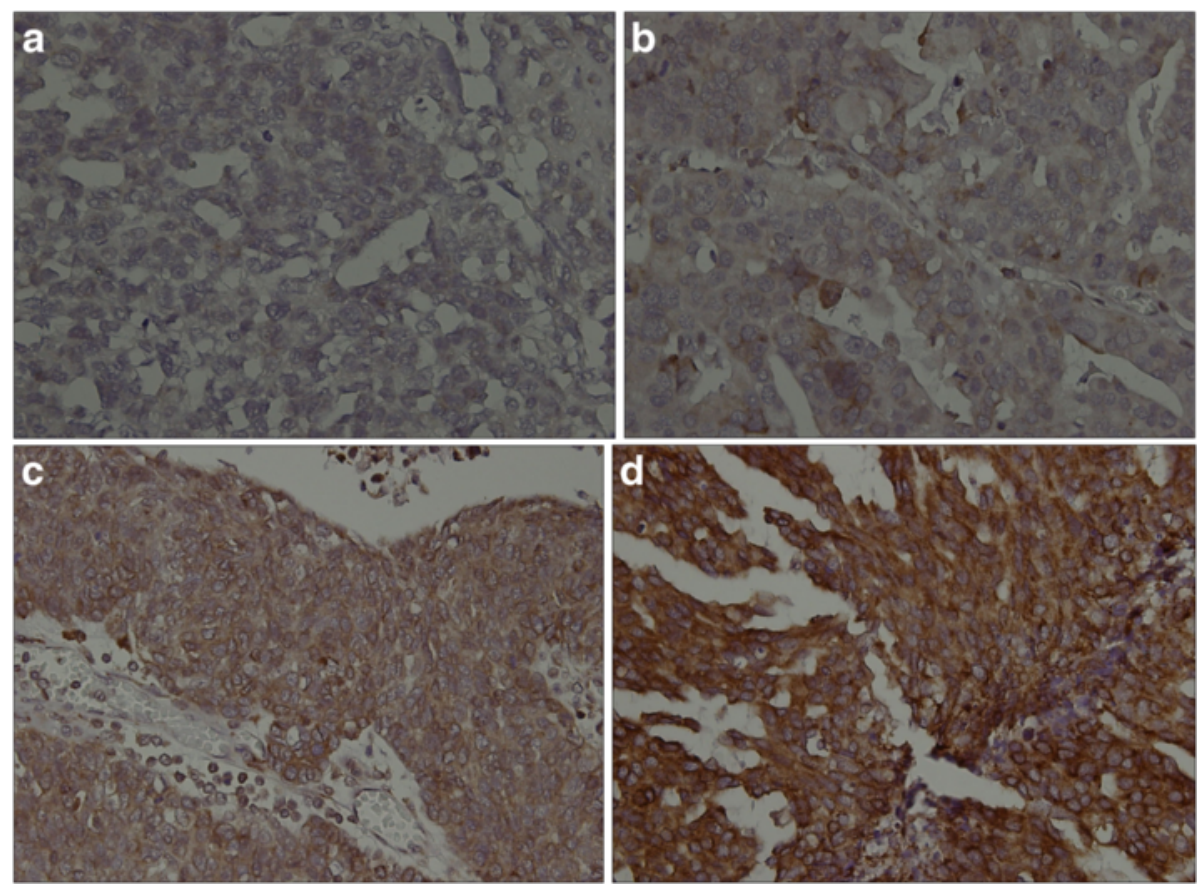

Fig. 1 Cytoplasmic immunoexpression of DEF6 in high-grade serous carcinoma. Case representatives show (a) negative (less than $5 \%$ ): score 0 ; (b) weak (5-25\%): score 1; (c) moderate (26-75\%): score 2; (d) strong (more than $75 \%$ ): score 3 of tumor cells

expression of p16 could be observed in $74.7 \%$ (56/75) of high-grade serous carcinomas, and commonly lost (up to $80.6 \%)$ in mucinous carcinomas. There was some variation in p16 staining in the endometrioid carcinoma and clear cell carcinoma groups. In summary, highgrade serous carcinoma showed the highest frequencies of increased DEF6 and p16 expressions, as well as aberrant p53 expression.

\section{Expression of DEF6 in ovarian carcinoma cell lines}

The expression profile of DEF6 was studied in several ovarian carcinoma cell lines by Western blot. Among six ovarian carcinoma cells examined, the expression of DEF6 in TOV-112D (endometrioid carcinoma) and OVCAR3 (serous carcinoma) cells were found to be relatively high when compared to other ovarian cells (Fig. 2). These findings were compatible with immunohistochemical results. This indicated that DEF6 was preferentially overexpressed in ovarian serous and endometrioid carcinomas. The level of DEF6 in A2780 (unknown histology subtype) and TOV-21G (clear cell carcinoma) was comparatively low among the six cells, however, they revealed a doubleband pattern on the DEF6 region as similar to the oral carcinoma cells.

\section{Prognostic values of co-expression DEF6/p16 and DEF6/p53}

We analyzed the prognostic significance of DEF6, p16 and p53 in the whole group of 180 cases of ovarian carcinoma. Four subgroups of patients each were formed according to DEF6/p16 and DEF6/p53 co-expressions (Table 2). The group with positive DEF6 and positive p16 expression was associated with the lowest OS $(P=0.027)$

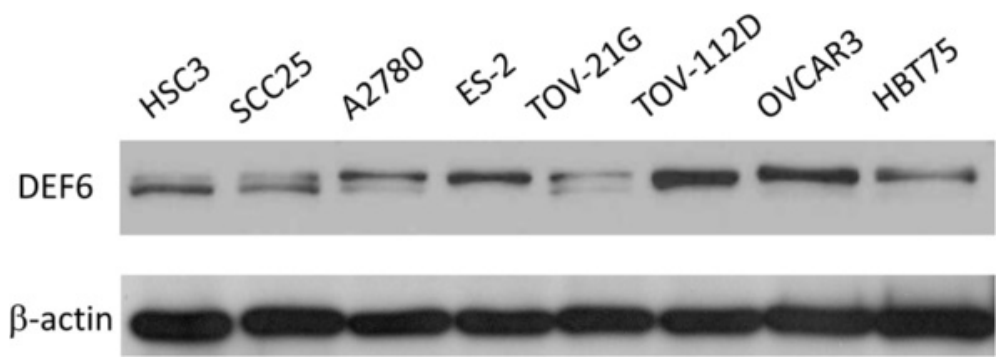

Fig. 2 Expression of DEF6 in ovarian carcinoma cell lines. The expression profile of DEF6 was studied in six ovarian carcinoma cell lines. A2780: unknown type; ES-2 and TOV-21G: clear cell carcinoma; TOV-112D: endometrioid carcinoma; OVCAR3 and HBT75: serous carcinoma. Two oral carcinoma cells, HSC-3 and SCC25, were included as positive controls. $\beta$-actin is detected as a loading control 
Table 2 Overall survival (OS) and disease-free survival (DFS) according to DEF6/p16 and DEF6/p53 co-expressions

\begin{tabular}{|c|c|c|c|c|c|c|c|c|}
\hline & OS (SE) & $95 \% \mathrm{Cl}$ & Median (SE) $(95 \%$ Cl) & $P$-value & DFS (SE) & $95 \% \mathrm{Cl}$ & Median (SE) (95\% Cl) & $P$-value \\
\hline \multicolumn{9}{|l|}{ DEF6/p16 co-expression } \\
\hline $\begin{array}{l}\text { DEF6-negative/ } \\
\text { p16-negative }\end{array}$ & $75(11)$ & $53-98$ & $\begin{array}{l}90(30) \\
30-149\end{array}$ & $0.027^{*}$ & $75(11)$ & $52-98$ & $\begin{array}{l}90(30) \\
30-149\end{array}$ & $0.023^{*}$ \\
\hline $\begin{array}{l}\text { DEF6-negative/ } \\
\text { p16-positive }\end{array}$ & $54(16)$ & $22-86$ & $\begin{array}{l}48(30) \\
0-107\end{array}$ & & $54(16)$ & $22-86$ & $\begin{array}{l}48(30) \\
0-107\end{array}$ & \\
\hline $\begin{array}{l}\text { DEF6-postive/ } \\
\text { p16-negative }\end{array}$ & $41(10)$ & $22-61$ & $\begin{array}{l}27(10) \\
6-47\end{array}$ & & $39(9)$ & $19-58$ & $\begin{array}{l}27(9) \\
8-45\end{array}$ & \\
\hline $\begin{array}{l}\text { DEF6-positive/ } \\
\text { p16-positive }\end{array}$ & $32(6)$ & $19-45$ & $\begin{array}{l}21(4) \\
12-30\end{array}$ & & $32(6)$ & $19-44$ & $\begin{array}{l}19(3) \\
12-25\end{array}$ & \\
\hline \multicolumn{9}{|l|}{ DEF6/p53 co-expression } \\
\hline $\begin{array}{l}\text { DEF6-negative } \\
\text { /p53 (scores } 1 \text { and 2) }\end{array}$ & $73(10)$ & $52-93$ & $\begin{array}{l}72(20) \\
31-113\end{array}$ & $0.031^{*}$ & $73(10)$ & $52-93$ & $\begin{array}{l}72(20) \\
31-113\end{array}$ & $0.028^{*}$ \\
\hline $\begin{array}{l}\text { DEF6-negative } \\
\text { /p53 (scores } 0 \text { and 3) }\end{array}$ & $64(17)$ & $30-99$ & $\begin{array}{l}76(79) \\
0-230\end{array}$ & & $64(17)$ & $30-97$ & $\begin{array}{l}76(79) \\
0-230\end{array}$ & \\
\hline $\begin{array}{l}\text { DEF6-postive } \\
\text { /p53 (scores } 1 \text { and 2) }\end{array}$ & $48(11)$ & $26-70$ & $\begin{array}{l}48(25) \\
0-97\end{array}$ & & $44(10)$ & $22-65$ & $\begin{array}{l}31(16) \\
0-62\end{array}$ & \\
\hline $\begin{array}{l}\text { DEF6-positive } \\
\text { /p53 (scores } 0 \text { and 3) }\end{array}$ & $29(6)$ & $16-41$ & $\begin{array}{l}19(2) \\
13-24\end{array}$ & & $28(6)$ & $16-41$ & $\begin{array}{l}17(1) \\
14-19\end{array}$ & \\
\hline
\end{tabular}

$\mathrm{SE}$, Standard error; $\mathrm{Cl}$, confidence interval

*significant difference

Log-rank test

DEF6 positive: Scores 2 and 3; DEF6 negative: Scores 0 and 1

p16 positive: Scores 2 and 3; p16 negative: Scores 0 and 1

and DFS $(P=0.023)$, whereas the DEF6 and p16 negative group had the highest OS and DFS. Similarly, the presence of aberrant p53 expression with concomitant DEF6 expression was statistically associated with worst prognosis, whereas the group negative for both had the best OS and DFS ( $P=0.031$ for OS and $P=0.028$ for DFS, respectively).

\section{Effects of DEF6, p16 and p53 expression on the patient's overall survivals}

Ovarian carcinoma patients with high DEF6 expression were associated with a poor overall survival compared with the patients with low DEF6 expression $(P=0.008$; Fig. 3a). Also, ovarian carcinoma patients with high 16 expression had lower overall survival rate than those with low p16 expression, as determined using the Kaplan-Meier method $(P=0.022$; Fig. 3b). Subsequently, we carried out survival analyses according to IHC evaluations in four different histological subtypes of ovarian carcinoma (see Additional file 1). Patients with aberrant p53 expression had shorter survival rate than those with insignificant p53 expression in high-grade serous carcinoma $(P=0.012)$. More importantly, in ovarian clear cell carcinoma, high expression of DEF6 was associated with shorter overall survival as compared to low DEF6 expression $(P=0.001$, Fig. 4$)$.

\section{Prognostic effects of clinicopathological factors and immunohistochemistry}

Univariate (Table 3) and multivariate (Table 4) analyses were conducted to analyze the clinicopathological and
IHC characteristics of the ovarian cancer patient cohorts to elucidate the crucial prognostic factors. Univariate analysis identified FIGO stage $(P=0.023$ for OS and $P=0.034$ for DFS), DEF6 ( $P=0.013$ for OS and $P=0.009$ for DFS) and p16 ( $P=0.026$ for OS and $P=0.031$ for DFS $)$ as prognostic factors. Upon multivariate analysis, strong DEF6 was the only independent prognostic factor correlated with shorted OS (HR 2.115; $P=0.025)$ and DFS (HR 2.248; $P=0.016)$.

\section{Discussion}

DEF6 is a conserved protein associated with the functions of lymphocytes and is highly expressed in T-cells and T-cell homing organs [4-27]. DEF6 was expressed in breast cancer cells [20], oral squamous cell carcinoma [21], colorectal carcinoma [19], and in tumor vessels of renal cell carcinoma [22]. The role of DEF6 expression in human cancer is unclear, and the prognostic significance of DEF6 expression and the co-expression of p16 and p53 in ovarian carcinomas are largely unknown.

In this study of 180 cases of ovarian carcinoma using immunohistochemistry, a high expression of DEF6 was commonly found in ovarian carcinomas, and associated with different histology subtypes, advanced FIGO stage, and reduced overall survival (OS) and disease free survival (DFS). Strong expression of DEF6 was observed in high-grade serous carcinoma and endometrioid carcinoma. These findings were supported by the high expression of DEF6 in two serous, two clear cell and one endometrioid carcinoma cell lines, suggestive of the 

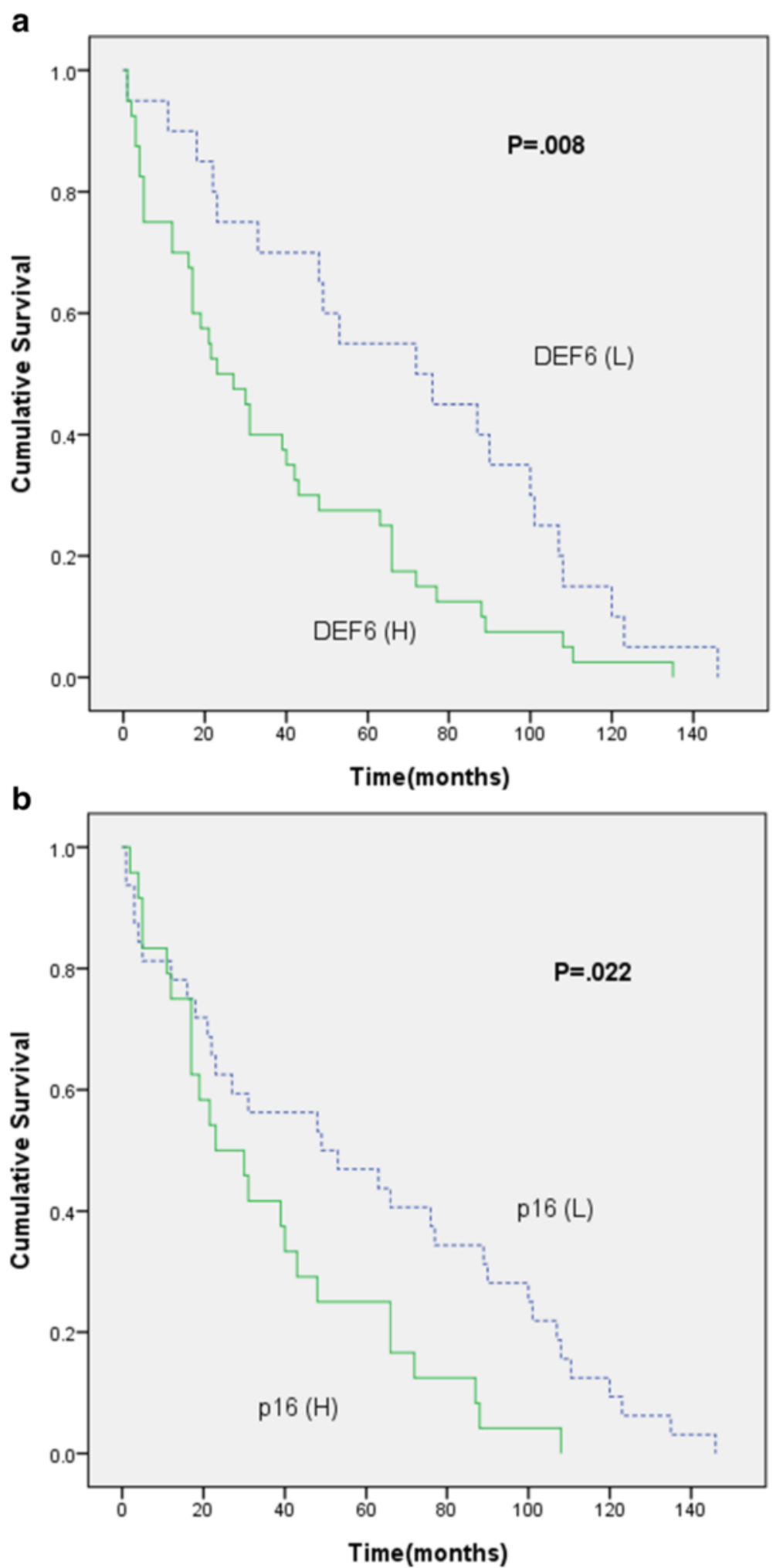

Fig. 3 Kaplan-Meier curves for overall survival according to protein expression levels in ovarian carcinomas $(n=180)$. a Patients with high DEF6 expression (scores 2 and $3 ; n=118$ ) versus low DEF6 expression (scores 0 and $1 ; n=62$ ). (H: high DEF6 expression; L: low DEF6 expression); (b) Patients with high p16 expression (scores 2 and $3 ; n=80$ ) versus low p16 expression (scores 0 and $1 ; n=97)$. (H: high p16 expression; L: low p16 expression) 


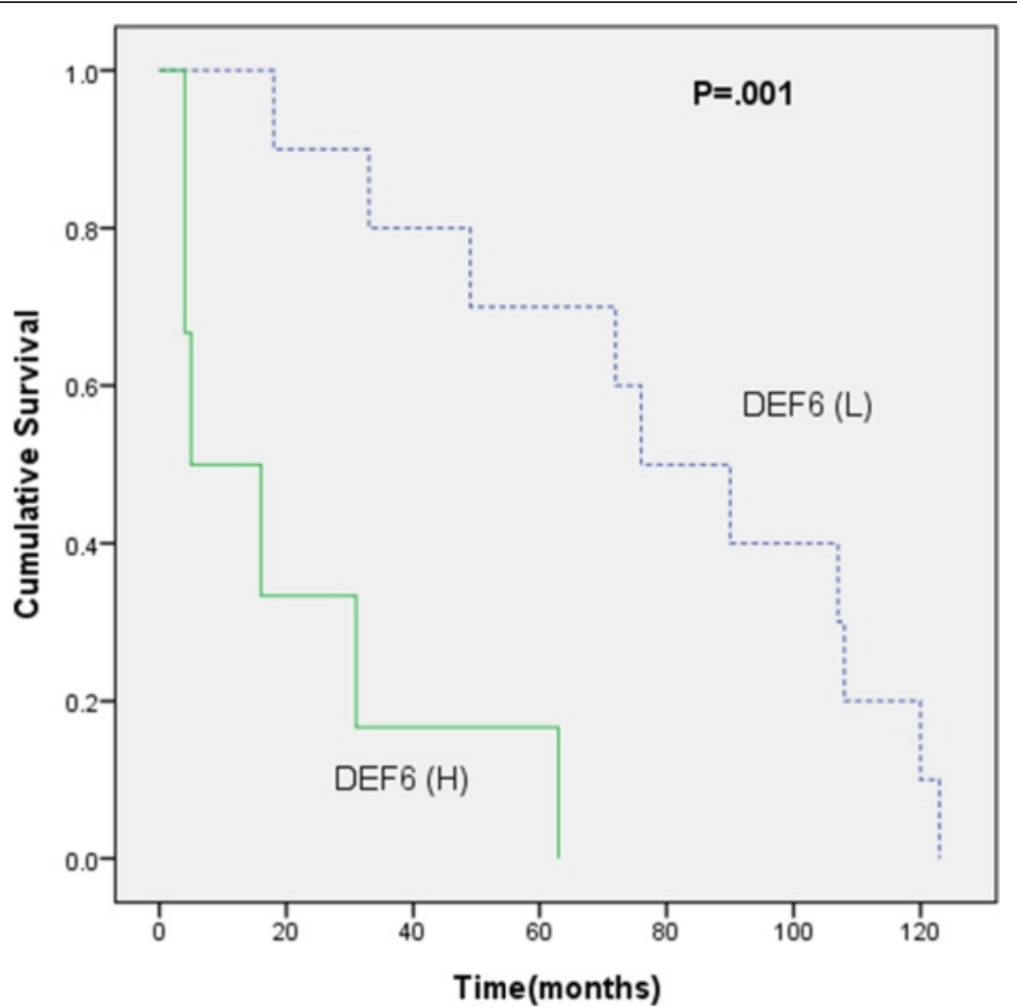

Fig. 4 Kaplan-Meier curves for overall survival in patients with high DEF6 expression (scores 2 and 3; $n=21$ ) versus low DEF6 expression (scores 0 and $1 ; n=20)$ in clear cell carcinoma. (H: high DEF6 expression; L: low DEF6 expression)

frequent expression of DEF6 in ovarian carcinoma tissues and cells.

There are many molecular markers possessing prognostic value. We compared the clinicopathological parameters and prognostic factors of DEF6, p16 and p53. The p16 is a cyclin-dependent kinase inhibitor which is integral to the retinoblastoma ( $\mathrm{Rb})$ genemediated control of the $G_{1}-S$ phase transition of the cell cycle [28]. It was also shown that ectopic expression of DEF6 shortened the $G_{1}$ interval in the cell cycle, and increased cyclin D1 expression [21]. It is therefore important to explore the cooperation between the two genes in the tumor progression. Although p16 has been widely regarded as a surrogate marker of high-risk human papillomavirus (HPV) in uterine cervical pathology, the role of p16 protein expression in ovarian carcinomas remains limited. In our study (Table 1), we found the highest percentage of score 3 p16 expression $(74.7 \%)$ in high-grade serous carcinoma, an ovarian carcinoma with notorious poor outcome, and similarly DEF6 (52\%). Our findings suggest that p16 is also a valuable prognostic marker for ovarian carcinomas. Recent studies have investigated the diagnostic role of p16 and prognostic indicator of p16 with clinical outcome in ovarian/tubal high-grade serous carcinoma. As p16 was expressed in the majority of p53positive and p53-negative serous tubal intraepithelial carcinomas, the addition of p16 helped to compensate the practical limitations of p53 in the diagnosis of serous tubal intraepithelial carcinomas [24]. Moreover, a recent study on ovarian/tubal high grade serous carcinomas revealed three distinct subgroups according to p16 expression and RB1 status (i.e., p16 homogenous stain/RB1, p16 homogenous stain/RB1+, and p16 heterogeneous stain/RB1+), which possessed clinical relevance for stage and patient outcome upon multivariate analysis [29]. We also assessed the prognostic significance of the status of DEF6/p16 co-expression. Notably, the co-expression of both proteins was associated closely with adverse clinical outcome (Table 2). The OS and DFS, in particular, were the shortest in the group with strong DEF6 and p16 coexpression, followed by DEF6+/p16- and DEF6-/p16+, while the DEF6-/p16- group had the longest survival rates ( $P=0.027$ for OS and $P=0.023$ for DFS, respectively). These findings suggest that DEF6 and p16 positively interact and contribute to the tumor progression in ovarian carcinoma, and the molecular mechanisms deserve to be further studied. This suggestion is supported by the observations that both proteins play important role in the control of the $G_{1}-S$ phase transition of the cell cycle $[21,28]$.

DEF6 is a novel p53 target gene and negatively regulated by $\mathrm{p} 53$, it can suppress cisplatin-mediated apoptosis of breast cancer cells [23]. In the present study, we 
Table 3 Univariate analyses showing HRs for patient OS and DFS conferred age, FIGO stage, histologic subtypes, DEF6, p53, p16 and ER expression $(N=180)$

\begin{tabular}{|c|c|c|c|c|c|c|c|}
\hline \multirow[t]{2}{*}{ Variables } & \multirow{2}{*}{$\begin{array}{l}\text { Total } \\
\text { No. }\end{array}$} & \multicolumn{3}{|c|}{ Overall survival (OS) } & \multicolumn{3}{|c|}{ Disease-free survival (DFS) } \\
\hline & & $\overline{\mathrm{HR}}$ & $95 \% \mathrm{Cl}$ & $P$-value & $\overline{\mathrm{HR}}$ & $95 \% \mathrm{Cl}$ & $P$-value \\
\hline Age, years & & & & 0.990 & & & 0.900 \\
\hline$\leqq 50$ & 72 & $1.00^{\mathrm{a}}$ & & & $1.00^{\mathrm{a}}$ & & \\
\hline$>50$ & 108 & 1.003 & $0.595-1.693$ & & 1.034 & $0.613-1.745$ & \\
\hline FIGO stage & & & & $0.023^{*}$ & & & $0.034^{*}$ \\
\hline 1 & 86 & $1.00^{\mathrm{a}}$ & & & $1.00^{\mathrm{a}}$ & & \\
\hline$\|$ & 23 & 1.141 & $0.518-2.511$ & & 1.119 & $0.509-2.460$ & \\
\hline III & 64 & 2.282 & $1.217-4.279$ & & 2.166 & $1.161-4.040$ & \\
\hline IV & 7 & 5.300 & $1.173-23.950$ & & 4.939 & $1.100-22.172$ & \\
\hline Histologic subtypes & & & & 0.303 & & & 0.284 \\
\hline Serous carcinoma & 75 & $1.00^{\mathrm{a}}$ & & & $1.00^{\mathrm{a}}$ & & \\
\hline Mucinous carcinoma & 36 & 0.529 & $0.247-1.135$ & & 0.531 & $0.247-1.138$ & \\
\hline Endometrioid carcinoma & 28 & 0.786 & $0.342-1.808$ & & 0.882 & $0.382-2.037$ & \\
\hline Clear cell carcinoma & 41 & 0.607 & $0.317-1.162$ & & 0.608 & $0.318-1.163$ & \\
\hline DEF6 & & & & $0.013^{*}$ & & & $0.009^{*}$ \\
\hline Scores 0 and 1 & 62 & $1.00^{\mathrm{a}}$ & & & $1.00^{\mathrm{a}}$ & & \\
\hline Scores 2 and 3 & 118 & 2.041 & $1.159-3.594$ & & 2.119 & $1.202-3.737$ & \\
\hline p16 & & & & $0.026^{*}$ & & & $0.031^{*}$ \\
\hline Scores 0 and 1 & 97 & $1.00^{\mathrm{a}}$ & & & $1.00^{\mathrm{a}}$ & & \\
\hline Scores 2 and 3 & 80 & 1.919 & $1.082-3.402$ & & 1.873 & $1.057-3.318$ & \\
\hline p53 & & & & 0.181 & & & 0.205 \\
\hline Scores 1 and 2 & 81 & $1.00^{\mathrm{a}}$ & & & $1.00^{\mathrm{a}}$ & & \\
\hline Scores 0 and 3 & 98 & 1.450 & $0.841-2.499$ & & 1.421 & $0.825-2.448$ & \\
\hline
\end{tabular}

aeference category for HR (Hazard Ratio) calculation with variable

$\mathrm{Cl}$ : Confidence Interval. (*Significant difference)

Table 4 Multivariate analyses showing HRs for patient OS and DFS conferred FIGO stage, DEF6 and p16 expression ( $N=180)$

\begin{tabular}{|c|c|c|c|c|c|c|}
\hline \multirow[t]{2}{*}{ Variable } & \multicolumn{3}{|c|}{ Overall survival (OS) } & \multicolumn{3}{|c|}{ Disease-free survival (DFS) } \\
\hline & $\mathrm{HR}$ & $95 \% \mathrm{Cl}$ & $P$-value & $\mathrm{HR}$ & $95 \% \mathrm{Cl}$ & $P$-value \\
\hline \multicolumn{7}{|l|}{ FIGO stage } \\
\hline I & $1.00^{\mathrm{a}}$ & & & $1.00^{\mathrm{a}}$ & & \\
\hline$\|$ & 0.624 & $0.235-1.658$ & 0.344 & 0.591 & $0.222-1.575$ & 0.293 \\
\hline III & 1.480 & $0.563-3.890$ & 0.426 & 1.373 & $0.522-3.609$ & 0.520 \\
\hline IV & 3.845 & $0.734-20.133$ & 0.111 & 3.496 & $0.671-18.206$ & 0.137 \\
\hline DEF6 & & & $0.025^{*}$ & & & $0.016^{*}$ \\
\hline Score 0 and 1 & $1.00^{\mathrm{a}}$ & & & $1.00^{\mathrm{a}}$ & & \\
\hline Score 2 and 3 & 2.115 & $1.097-4.079$ & & 2.248 & $1.161-4.352$ & \\
\hline p16 & & & 0.611 & & & 0.611 \\
\hline Score 0,1 and 2 & $1.00^{\mathrm{a}}$ & & & $1.00^{\mathrm{a}}$ & & \\
\hline Score 3 & 1.250 & $0.530-2.945$ & & 1.250 & $0.529-2.953$ & \\
\hline
\end{tabular}

aeference category for HR (Hazard Ratio) calculation with variable

$\mathrm{Cl}$ : Confidence Interval. (*Significant difference) 
found that strong DEF6 expression and aberrant p53 expression had the shortest survival, whereas coexpression of insignificant p53 expression and negative DEF6 displayed the longest survival rate, with the other two groups in between $(P=0.031$ for OS and $P=0.028$ for DFS, Table 3). Thus, the evaluation of the p53 status coupled with DEF6 might be important for risk stratification, which certainly awaits sequencing validation of the p53 status for further clarification.

We further evaluated the overall survival rates of DEF6, p16 and p53 in all patients and four different histology subtypes of ovarian carcinoma by using Kaplan-Meier analysis. Significantly shorter survival rates were seen in patients with high expressions of DEF6 $(P=0.008)$ and p16 $(P=0.022)$. Patients with aberrant p53 expression in high-grade serous carcinoma had shorter overall survival $(P=0.012)$. Surprisingly, high expression of DEF6 in clear cell carcinoma was significantly correlated to shorter overall survival $(P=0.001)$. We enrolled in total 41 cases of ovarian clear cell carcinoma (30 FIGO stage I, 4 FIGO stage II and 7 FIGO stage III) in this study. Therefore, our study suggests that patients of ovarian clear cell carcinoma with high DEF6 expression deserve a poor prognostic factor compared with other histological subtypes. Our study is the first report on the prognostic impact of DEF6 overexpression in early-stage ovarian clear cell carcinomas, however in-depth investigations are necessary.

Univariate analysis showed that FIGO stage, DEF6 and p16 were associated with shorter survival (Table 3). Upon multivariate analysis, DEF6 remained the significant prognostic value (Table 4). These findings suggest that DEF6 in ovarian carcinomas may facilitate tumor cell growth or proliferation, motility, invasion and metastasis, leading to high tumor stage and hence poor prognosis.

\section{Conclusions}

DEF6 was often overexpressed in ovarian carcinomas, particularly in high-grade serous carcinoma and endometrioid carcinoma cells and tissues. DEF6 overexpression in early-stage ovarian clear cell carcinomas may have potential role as a poor prognostic factor. Multivariate analysis showed that DEF6 might serve as independent prognostic biomarker. Our findings suggest that DEF6 contribute cooperatively with p16 and p53, another two important cell cycle regulators, toward high tumor stage and poor OS and DFS in ovarian carcinomas. Hence DEF6 expression deserves further investigation especially its potential as therapeutic target in the dreadful ovarian carcinomas.

\section{Additional file}

Additional file 1: Figure S1. Kaplan-Meier curves for overall survival in four histological subtypes of ovarian carcinoma patients according to DEF6, p16 and p53 expressions. A, Patients with high DEF6 expression (scores 2 and $3 ; n=60$ ) versus low DEF6 expression (scores 0 and $1: n=15$ ) in highgrade serous carcinoma. (H: high DEF6 expression; L: low DEF6 expression). B, Patients with high p16 expression (scores 2 and $3 ; n=56$ ) versus low p16 expression (scores 0 and $1 ; n=18$ ) in high-grade serous carcinoma. (H: high p16 expression; L: low p16 expression). C, Patients with aberrant p53 expression (scores 0 and $3 ; n=62$ ) versus insignificant p53 expression (scores 1 and $2 ; n=12$ ) in high-grade serous carcinoma. D, Patients with high DEF6 expression (scores 2 and $3 ; n=20$ ) versus low DEF6 expression (scores 0 and $1 ; n=16$ ) in mucinous carcinoma. (H: high DEF6 expression; L: low DEF6 expression). E, Patients with high p16 expression (scores 2 and $3 ; n=32$ ) versus low p16 expression (scores 0 and $1 ; n=3$ ) in mucinous carcinoma. (H: high p16 expression; L: low p16 expression). F, Patients with aberrant p53 expression (scores 0 and $3 ; n=17$ ) versus insignificant p53 expression (scores 1 and $2 ; n=19$ ) in mucinous carcinoma. G, Patients with high DEF6 expression (scores 2 and $3 ; n=6$ ) versus low DEF6 expression (scores 0 and $1 ; n=22$ ) in endometrioid carcinoma. (H: high DEF6 expression; L: low DEF6 expression). $\mathrm{H}$, Patients with high p16 expression (scores 2 and $3 ; n=17$ ) versus low p16 expression (scores 0 and $1 ; n=11$ ) in endometrioid carcinoma. (H: high p16 expression; L: low p16 expression). I, Patients with aberrant p53 expression (scores 0 and $3 ; n=9$ ) versus insignificant p53 expression (scores 1 and 2; $n=19$ ) in endometrioid carcinoma. J, Patients with high p16 expression (scores 2 and $3 ; n=30$ ) versus low p16 expression (scores 0 and $1 ; n=11$ ) in clear cell carcinoma. (H: high p16 expression; L: low p16 expression). K, Patients with aberrant p53 expression (scores 0 and $3 ; n=10$ ) versus insignificant p53 expression (scores 1 and $2 ; n=31$ ) in clear cell carcinoma. (ZIP $195 \mathrm{~kb})$

\section{Abbreviations}

DFS, disease-free survival; FIGO, International Federation of Gynecology and Obstetrics; H\&E, hematoxylin and eosin; HR, Hazard Ratio; IHC, immunohistochemical; IBP, interferon regulatory factor 4 binding protein; OS, overall survival; TMA, tissue microarray

\section{Acknowledgements}

The authors thank Professor Hey-Chi Hsu for his assistance and critical review. We thank Professor Chao-Lien Liu at the Department of Medical Laboratory Science and Biotechnology, Taipei Medical University for providing the lysates of OVCAR3 and HBT75 cells.

\section{Funding}

This work was funded by a grant from Health and Welfare Surcharge of Tobacco Products (DOH102-TD-C-111-008) from Ministry of Health and Welfare to Center of Excellence for Cancer Research of Taipei Medical University (to C-L Chen) and Grant 102TMU-SHH-21 from the Shuang Ho Hospital, Taipei Medical University (to P-L Liew).

\section{Availability of data and materials}

All measurements were collected and recorded in Microsoft Excel. Both Dr. Phui-Ly Liew and Dr. Chi-Long Chen have copies of these documents. The clinical materials are H\&E and immunohistochemical slides which are stored in the Surgical Pathology archives at Taipei Medical University. All material is available upon request.

\section{Authors' contributions}

CYF, YCL and PLL are responsible for laboratory work, sample collection and data analysis. YCL is responsible for data collection and clinical data analysis. $\mathrm{CLC}$ and JSC are responsible for project design. PLL is responsible for manuscript preparation. All authors read and approved the final manuscript.

\section{Competing interests}

The authors declare that they have no competing interests.

\section{Consent for publication}

Not applicable.

\section{Ethics approval and consent to participate}

The study was approved by the Institutional Review Board of Taipei Medical University (TMU-IRB 99049). 


\section{Author details}

'Department of Pathology, Shuang Ho Hospital, Taipei Medical University, New Taipei City 23561, Taiwan. ${ }^{2}$ Department of Pathology, School of Medicine, College of Medicine, Taipei Medical University, No. 250, Wu Xing Street, Taipei 11031, Taiwan. ${ }^{3}$ Department of Pathology, Wan Fang Hospital Taipei Medical University, Taipei 116, Taiwan. ${ }^{4}$ Graduate Institute of Medical Sciences, College of Medicine, Taipei Medical University, Taipei 11031, Taiwan. ${ }^{5}$ Department of International Business, Chien Hsin University of Science and Technology, Taoyuan 32097, Taiwan. 'Department of Pathology, Taipei Medical University Hospital, Taipei Medical University, Taipei 11031 , Taiwan.

Received: 9 May 2016 Accepted: 19 July 2016

Published online: 03 August 2016

\section{References}

1. Kurman RJ, Carcangiu ML, Herrington CS, Young RH. World Health Organization Classification of Tumours of Female Reproductive Organs $4^{\text {th }}$ ed. Lyon: IARC Press; 2014.

2. Clark TG, Stewart ME, Altman DG, Gabra H, Smyth JF. A prognostic model for ovarian cancer. Br J Cancer. 2001;85:944-52.

3. Cannistra SA. Cancer of the ovary. N Engl J Med. 2004:351:2519-29.

4. Gupta S, Lee A, Hu C, Fanzo J, Goldberg I, Cattoretti G, et al. Molecular cloning of IBP, a SWAP-70 homologous GEF, which is highly expressed in the immune system. Hum Immunol. 2003;64:389-401.

5. Biswas PS, Gupta S, Stirzaker RA, Kumar V, Jessberger R, Lu TT, et al. Dual regulation of IRF4 function in T and $B$ cells is required for the coordination of T-B cell interactions and the prevention of autoimmunity. J Exp Med. 2012;209:581-96.

6. Canonigo-Balancio AJ, Fos C, Prod'homme T, Bécart S, Altman A. SLAT/Def6 plays a critical role in the development of Th17 cell-mediated experimental autoimmune encephalomyelitis. J Immunol. 2009;183:7259-67.

7. Mehta H, Glogauer M, Bécart S, Altman A, Coggeshall KM. Adaptor protein SLAT modulates Fcgamma receptor-mediated phagocytosis in murine macrophages. J Biol Chem. 2009;284:11882-91.

8. Pernis AB. Rho GTPase-mediated pathways in mature CD4+ T cells. J Leukoc Biol. 2009;85:539-43.

9. Fanzo JC, Yang W, Jang SY, Gupta S, Chen Q, Siddiq A, et al. Loss of IRF-4-binding protein leads to the spontaneous development of systemic autoimmunity. J Clin Invest. 2006;116:703-14

10. Mavrakis KJ, McKinlay KJ, Jones P, Sablitzky F. DEF6, a novel PH-DH-like domain protein, is an upstream activator of the Rho GTPases Rac1, Cdc42, and RhoA. Exp Cell Res. 2004;294:335-44.

11. Xu X, Shuen WH, Chen C, Goudevenou K, Jones P, Sablitzky F. Swap70b is required for convergent and extension cell movement during zebrafish gastrulation linking Wnt11 signalling and RhoA effector function. Dev Biol. 2014:386:191-203.

12. Hey F, Czyzewicz N, Jones P, Sablitzky F. DEF6, a novel substrate for the Tec kinase ITK, contains a glutamine-rich aggregation-prone region and forms cytoplasmic granules that co-localize with P-bodies. J Biol Chem. 2012;287: 31073-84.

13. Goudevenou K, Martin P, Yeh YJ, Jones P, Sablitzky F. Def6 is required for convergent extension movements during zebrafish gastrulation downstream of Wnt5b signaling. PLoS ONE. 2011;6:e26548.

14. Feau S, Schoenberger SP, Altman A, Bécart S. SLAT regulates CD8+ T cell clonal expansion in a Cdc42- and NFAT1-dependent manner. J Immunol. 2013;190:174-83.

15. Chen Q, Gupta S, Pernis AB. Regulation of TLR4-mediated signaling by IBP/ Def6, a novel activator of Rho GTPases. Autoimmun Rev. 2009:8:199-203.

16. Samson T, Will C, Knoblauch A, Sharek L, von der Mark K, Burridge K, et al. Def6 , a guanine nucleotide exchange factor for Rac1, interacts with the skeletal muscle integrin chain alpha7A and influences myoblast differentiation. J Biol Chem. 2007;282:15730-42.

17. Oka T, Ihara S, Fukui Y. Cooperation of DEF6 with activated Rac in regulating cell morphology. J Biol Chem. 2007;282:2011-8.

18. Subramanian S, West RB, Marinelli RJ, Nielsen TO, Rubin BP, Goldblum JR, et al. The gene expression profile of extraskeletal myxoid chondrosarcoma. J Pathol. 2005:206:433-44.

19. Zhang Z, Wang Q, Li P, Zhou Y, Li S, Yi W, et al. Overexpression of the Interferon regulatory factor 4-binding protein in human colorectal cancer and its clinical significance. Cancer Epidemiol. 2009;33:130-6.
20. Li P, Zhang Z, Wang Q, Li S, Zhang Y, Bian X, et al. The ectopic expression of IFN regulatory factor 4-binding protein is correlated with the malignant behavior of human breast cancer cells. Int Immunopharmacol. 2009;9:1002-9.

21. Jian CX, Yang MZ, Li P, Xiong J, Zhang ZJ, Li CJ, et al. Ectopically expressed IBP promotes cell proliferation in oral squamous cell carcinoma. Cancer Invest. 2012;30:748-56.

22. Otsubo T, Hida Y, Ohga N, Sato H, Kai T, Matsuki Y, et al. Identification of novel targets for antiangiogenic therapy by comparing the gene expressions of tumor and normal endothelial cells. Cancer Sci. 2014;105:560-7.

23. Yang M, Yuan F, Li P, Chen Z, Chen A, Li S, et al. Interferon regulatory factor 4 binding protein is a novel p53 target gene and suppresses cisplatininduced apoptosis of breast cancer cells. Mol Cancer. 2012;13:11-54.

24. Novak M, Lester J, Karst AM, Parkash V, Hirsch MS, Crum CP, et al. Stathmin 1 and p16INK4A are sensitive adjunct biomarkers for serous tubal intraepithelial carcinoma. Gynecol Oncol. 2015:139:104-11.

25. O'Neill CJ, McBride HA, Connolly LE, Deavers MT, Malpica A, McCluggage WG. High-grade ovarian serous carcinoma exhibits significantly higher p16 expression than low-grade serous carcinoma and serous borderline tumour. Histopathology. 2007:50:773-9.

26. Liew PL, Hsu CS, Liu WM, Lee YC, Lee YC, Chen CL. Prognostic and predictive values of Nrf2, Keap1, p16 and E-cadherin expression in ovarian epithelial carcinoma. Int J Clin Exp Pathol. 2015:8:5642-9.

27. Becart S, Charvet C, Ann J, Balancio C, De Trez C, Tanaka Y, et al. SLAT regulates Th1 and Th2 inflammatory responses by controlling Ca2+/NFAT signaling. J Clin Invest. 2007;117:2164-75.

28. Sharpless NE. INK4a/ARF: a multifunctional tumor suppressor locus. Mutat Res. 2005;576:22-38.

29. Beirne JP, McArt DG, James JA, Salto-Tellez M, Maxwell P, McCluggage WG. p16 As a prognostic indicator in ovarian/tubal high grade serous carcinoma. Histopathology. 2016;68:615-8.

\section{Submit your next manuscript to BioMed Central} and we will help you at every step:

- We accept pre-submission inquiries

- Our selector tool helps you to find the most relevant journal

- We provide round the clock customer support

- Convenient online submission

- Thorough peer review

- Inclusion in PubMed and all major indexing services

- Maximum visibility for your research

Submit your manuscript at www biomedcentral com/submit
C BioMed Central 